\title{
Ueber die Einwirkung von Phenolen auf halogensubstituirte Fettsäuren;
}

\author{
von
}

\section{Dr. Ludwig Saarbach.}

Die Einwirkung von Phenol auf Monochloressigsäure hat W. Heintz ${ }^{1}$ ) zum Gegenstand einer eingehenden Untersuchung gemacht, welche einen Theil seiner grossen Arbeit: "Ueber zwei neue Reihen organischer Säuren" bildet. Am Schlusse der Abhandlung, in welcher er die Bildung der Oxacetsäure, Methoxacetsäure, Aethoxacetsäure, Amoxacetsäure und Phenoxacetsäure aus Monochloressigsäure bei Behandlung mit Natronhydrat, Natrium-Methylat, -Aethylat, -Amylat und -Phenylat beschreibt, spricht Heintz die Vermuthung aus, dass auch die übrigen Alkohole der Methylund Phenylreihe, sobald sie dem Natriummethylat entsprechende Natriumverbindungen zu bilden im Stande seien, sich zu der Monochloressigsäure vielleicht in ähnlicher Weise verhalten.

An der Lösung dieser Frage für die Phenylreihe wurde bis zur jüngsten Zeit nicht gearbeitet, erst in diesem Jahre unterzog Fritzsche ${ }^{2}$ ) die von Heintz schon dargestellte Phenoxacetsäure einer eingehenderen Untersuchung. Er änderte das Darstellungsverfahren für diese Säure, welche er Oxyphenylessigsäure nennt, in Bezug auf Ausbeute und Reinheit der Substanz mit bestem Erfolge darin ab, dass er Phenolnatrium, statt auf Monochloressigsäure, auf das Natronsalz derselben einwirken liess. Die Säure bildet sich nach folgender Gleichung:

$$
\mathrm{C}\left\{\begin{array}{l}
\mathrm{H}_{2} \\
\mathrm{Cl}
\end{array}\right\} \mathrm{COONa}+\mathrm{C}_{6} \mathrm{H}_{5} \mathrm{ONa}=\mathrm{NaCl}+\mathrm{C}\left\{\begin{array}{l}
\underset{\mathrm{H}_{6} \mathrm{H}_{5}}{\mathrm{O}_{5}} \\
\mathrm{C}
\end{array}\right\} \mathrm{COONa}
$$

1) Pogg. Ann. 109, 489.

$\left.{ }^{2}\right)$ Dies. Journ. [2] 19, 34; 20, 267. 
Diese Gleichung zu Grunde legend, untersuchte ich, ob erstens andere Phenole auf Monochloressigsäure in analoger Weise einwirken, und ob zweitens andere halogensubstituirte Säuren von Phenol in derselben Art angegriffen werden. Ich prüfte das Verhalten des Thymols, Eugenols und Orcins gegen Monochloressigsäure und andererseits des Phenols gegen $\alpha$.Monochlorpropionsäure und Dibrombernsteinsäure.

Vor wenigen Monaten veröffentlichte Giacosa ${ }^{1}$ ) eine Abhandlung, in welcher er die Einwirkung der Pyrogallussäure auf Monochloressigsäure, die Bildung der Pyrogalloltriglycolsäure, $\begin{aligned} \mathrm{C}_{6} \mathrm{H}_{3} & \text { O. O. } \mathrm{CH}_{2} \mathrm{COOH} \\ & \text { O. } \mathrm{CH}_{2} \mathrm{COOH} \text {, beschreibt. Giacosa's }\end{aligned}$ Darstellungsweise dieser Säure und die der Oxyphenylessigsäure durch Erhitzen von Pyrogallol resp. Phenol mit Monochloressigsäure unter allmählichem Zusetzen von Natronlauge wandte ich zur Gewinnung einiger von mir dargestellten Säuren an.

\section{Einwirkung von Phenol auf $\alpha$-Monochlorpropionsäure.}

25 Grm. $\alpha$-Chlorpropionsäure, nach der Buchanan'schen ${ }^{2}$ ) Vorschrift durch Destillation einer Mischung von milchsaurem Kalk und Fünffach-Chlorphosphor, Zersetzung des gewonnenen Chlorids mit Wasser und Destillation des Productes erhalten, werden mit einer concentrirten wässrigen Lösung von kohlensaurem Natron neutralisirt. $\mathrm{Zu}$ dem so entstandenen Natronsalz setzt man 24 Grm. möglichst trocknen und farblosen Phenolnatriums. Schon in der Kälte tritt eine Reaction ein, zu deren Beendigung man die Flüssigkeit auf dem Wasserbade in einer Porcellanschale bis zur breiigen Consistenz eindampft. Löst man diese Masse in Wasser und fügt Salzsäure zu, so fällt die neue Säure in krystallischen Klümpchen aus, welche, auf's Saugfilter gebracht, einige Male mit kaltem Wasser ausgewaschen und dann aus

1) Dies. Journ. [2] 19, 396 .

$\left.{ }^{2}\right)$ Anm. Chem. Pharm. 148, 170. 
heissem Wasser umkrystallisirt werden. Beim Erkalten der Lösung scheidet sich die Säure in langen, glasglänzenden Nadeln aus, welche, im Vacuum getrocknet und analysirt, folgende Werthe gaben:

I. $0,095 \mathrm{Grm}$. Substanz, mit Kupferoxyd verbrannt, lieferten 0,053 Grm. $\mathrm{H}_{2} \mathrm{O}$ und 0,226 Grm. $\mathrm{CO}_{2}$.

II. 0,150 Grm. Substanz lieferten, auf dieselbe Weise rerbrannt, $0,082 \mathrm{Grm} . \mathrm{H}_{2} \mathrm{O}$ und $0,358 \mathrm{Grm} . \mathrm{CO}_{2}$.

Oxyphenylpropionsäure, $\mathrm{CH}_{3} . \mathrm{C}\left\{\begin{array}{c}\mathrm{H} \\ \mathrm{OC}_{6} \mathrm{H}_{5}\end{array}\right\} \mathrm{COOH}$,

$\overbrace{\mathrm{C}_{9}=108=65,06 \%}$
$\mathrm{H}_{10}=10=6,03 \%$
$\mathrm{O}_{3}=\frac{48=28,91 \%}{166}=100,00$

gefunden:

I. II.

$64,88 \quad 65,09$

$6,19 \quad 6,07$

Die Entstehung der Säure erfolgt nach der Gleichung: $\mathrm{CH}_{3} \cdot \mathrm{C}\left\{\begin{array}{c}\mathrm{H} \\ \mathrm{Cl}\end{array}\right\} \mathrm{COONa}+\mathrm{C}_{6} \mathrm{H}_{5} \mathrm{ONa}=\mathrm{NaCl}+\mathrm{CH}_{3} . \mathrm{C}\left\{\underset{\underset{\mathrm{O}_{6} \mathrm{H}_{5}}{\mathrm{H}}}{\mathrm{C}}\right\} \mathrm{COONa}$.

- Die Oxyphenylpropionsäure ist kaum löslich in kaltem Wasser, in heissem schmilat sie, mit Wasserdämpfen ist sie fliichtig; beim Umkrystallisiren aus Wasser ist es deshalb rathsam, recht viel Lösungsmittel anzuwenden. Leicht löslich ist die Säure in Alkohol, Aether und in heissem Wasser. Ihr Schmelzpunkt liegt bei $112^{\circ}-113^{\circ}$.

In der wässrigen Lösung erzengt Eisenchlorid einen gelblichen Niederschlag.

Das eingangs dieser Arbeit erwähnte Giacosa'sche Verfahren zur Gewinnung dieser Säuren konnte ich, da mir keine $\alpha$-Chlorpropionsäure mehr zu Gebote stand, für die Darstellung der Oxyphenylpropionsäure nicht der Untersuchung unterziehen.

Salze der Oxyphenylpropionsäure.

Die Salze der Oxyphenylpropionsäure stellt man wegen der Flüchtigkeit der Säure mit Wasserdämpfen an besten durch Zusatz von Säure zu den in heissem Wasser gelösten 


\section{Saarbach: Einwirkung von Phenolen}

oder suspendirten kohlensauren Salzen dar; sie sind sämmtlich in Wasser löslich, besonders leicht die Alkalisalze.

Das Natronsalz, $\mathrm{CH}_{3} . \mathrm{C}\left\{\begin{array}{c}\mathrm{H} \\ \mathrm{OC}_{6} \mathrm{H}_{5}\end{array}\right\}$ COONa, schiesst beim Abdampfen in concentrisch gruppirten Nadeln an, welche sich in Wasser unter lebhaft rotirender Bewegung wieder lösen; in Alkohol ist es schwer löslich. Da das Salz schon beim Stehen über Schwefelsäure Wasser verliert, an der Luft aber sehr schnell zerfiliesst, konnte ich bei der Bestimmung des Wassergehaltes keine übereinstimmenden Resultate erhalten.

$0,3406 \mathrm{Grm}$. des Salzes gaben beim Abrauchen mit Schwefelsäure $0,1257 \mathrm{Grm}$. $\mathrm{SO}_{4} \mathrm{Na}_{2}$, entsprechend $11,95 \% \mathrm{Na}$ (ber. 11,23\%).

Eisenchlorid bewirkt in der wässrigen Lösung des Natronsalzes einen gelbbraunen flockigen Niederschlag, welcher sich beim Kochen in eine lebhaft blutrothe zähe Flüssigkeit umwandelt; beim Erwärmen mit Alkohol scheidet diese wieder gelbe Flocken ab.

Oxyphenylpropionsaures Kali krystallisirt in langen, sternförmig gruppirten Nadeln, welche beim Trocknen bis $130^{\circ} 1 \frac{1}{2}$ Moleküle $\mathrm{H}_{2} \mathrm{O}$ verlieren; bei weiterem Erhitzen schmelzen sie und bilden dann beim Erkalten eine glasähnliche Masse.

I. 0,1488 Grm. lufttrockner Substanz verloren, bis $130^{\circ}$ erhitzt, $0,0170 \mathrm{Grm} . \mathrm{H}_{2} \mathrm{O}$. $\mathrm{SO}_{4} \mathrm{~K}_{2}$.

II. 0,3604 Grm. gaben, mit $\mathrm{SO}_{4} \mathrm{H}_{2}$ abgeraucht, 0,1363 Grm.

$$
\begin{aligned}
& \mathrm{CH}_{3} \cdot \mathrm{C}\left\{\begin{array}{c}
\mathrm{H} \\
\mathrm{OC}_{6} \mathrm{H}_{5}
\end{array}\right\} \mathrm{COOK}+1 \frac{1 / 2}{\mathrm{H}_{2} \mathrm{O}} \\
& \text { verlangt: } \\
& 1{ }^{1}{ }_{2} \mathrm{H}_{2} \mathrm{O}=11,69 \% \\
& \mathrm{~K}=16,89 \text {, }
\end{aligned}
$$

Oxyphenylpropionsaurer Kalk. Durch Zersetzen von kohlensaurem Kalk mit Oxyphenylpropionsäure erhält man das Kalksalz der letzteren in kurzen wasserklaren Säulen, welche sich zu schön ausgebildeten, kugelförmigen Concretionen vereinigen. 
I. $0,0970 \mathrm{Grm}$. lufttrocknes Salz verloren bei $130^{\circ} 0,0087 \mathrm{Grm} . \mathrm{H}_{2} \mathrm{O}$.

II. 0,0883 Grm. entwässerte Substanz gaben, mit $\mathrm{SO}_{4} \mathrm{H}_{2}$ abgeraucht, $0,0330 \mathrm{Grm} . \mathrm{SO}_{4} \mathrm{Ca}$.

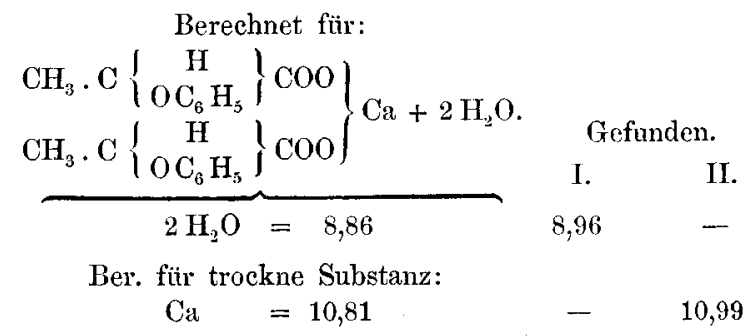

In Alkohol ist das Salz sehr schwer löslich.

Das Silbersalz, $\mathrm{CH}_{3} . \mathrm{C}\left\{\underset{\mathrm{OC}_{6} \mathrm{H}_{5}}{\mathrm{H}}\right\} \mathrm{COOAg}$, wird bei Zusatz von salpetersaurem Silber zur Lösung des Natronsalzes in kleinen, spitzen Nadeln erhalten, welche am Licht sich schnell schwärzen.

Das Kupfersalz fällt beim Zufügen von Kupfervitriollösung zur Lösung des oxyphenylpropionsauren Natrons als lebhaft hellgrün gefärbtes Pulver aus. Da das Salz schon unter Wasser schmilzt, so muss man bei seiner Darstellung Erwärmung sorgfältig vermeiden.

\section{Oxyphenylpropionsäure-Aethyläther.}

Schon bei längerem Stehen einer alkoholischen Lösung der Oxyphenylpropionsäure bildet sich ihr Aethyläther; am leichtesten gewinnt man ihn, wenn man durch die am Rückflusskühler im Wasserbad erhitzte alkoholische Lösung der Säure Salzsäure leitet. Das gewonnene Product wird in Wasser gegossen, wobei sich der Aether als ölige Schicht am Boden absetzt, welche, nach mehrmaligem Waschen mit kaltem Wasser, mit Wasserdämpfen destillirt wird. Der Aether geht dabei als ganz klare Flüssigkeit über; nach dem Trocknen mit Chlorcalcium analysirt, ergab er folgende Zahlen:

I. 0,1876 Grm. Substanz lieferten 0,1241 Grm. $\mathrm{H}_{2} \mathrm{O}$ und 0,4657 Grm. $\mathrm{CO}_{2}$.

II. $0,1953 \mathrm{Grm}$. lieferten $0,1291 \mathrm{Grm} . \mathrm{H}_{2} \mathrm{O}$ und $0,4845 \mathrm{Grm} . \mathrm{CO}_{2}$ 
Oxyphenylpropionsïure-Aethylïther: $\mathrm{CH}_{3} . \mathrm{C}\left\{\underset{\mathrm{O} \mathrm{C}_{0} \mathrm{H}_{5}}{\mathrm{H}}\right\}_{\mathrm{COOC}} \mathrm{H}_{5}$

$$
\begin{aligned}
& \text { entliält: } \\
& \mathrm{C}_{11}=132=68,04 \% \\
& \mathrm{H}_{14}=14=7,22, \\
& \mathrm{O}_{3}=48=24,74, \\
& 194 \quad 100,00
\end{aligned}
$$
gefunden:

I. 67,70 7,35 67,66 7,34

Er hat einen angenehmen, schwach chlorcoformähnlichen Geruch, siedet unzersetzt bei $243^{\circ}-244^{\circ}$. Sein specifisches Gewicht beträgt 1,360 bei $17,5^{n}$.

\section{Oxyphenylpropionsäure-Amid.}

Lässt man Oxyphenylpropionsäureäther einige Zeit mit überschüssigem wässrigem Ammoniak stehen, so setzen sich bald ziemlich grosse wasserklare Krystalle ab, welche, bei $120^{\circ}$ getrocknet, der Analyse unterworfen wurden: $\left.\mathrm{T} .=19^{\circ}\right)$.

0,2835 Grm. Substanz gaben 21 Cem. Stickstoff (Bar. = $753 \mathrm{Mm}$.,

Das Amid $\mathrm{C}_{9} \mathrm{H}_{11} \mathrm{O}_{2} \mathrm{~N}$

$$
\begin{array}{ll}
\text { enthält } & 8,49 \% \mathrm{~N}, \\
\text { gefunden } & 8,43 \% \mathrm{~N} .
\end{array}
$$

Seine Bildung geht glatt vor sich nach der Gleichung:

$$
\begin{aligned}
& \mathrm{CH}_{3}, \mathrm{C}\left\{\underset{\mathrm{OC}_{6} \mathrm{H}_{5}}{\mathrm{H}}\right\} \mathrm{COOC}_{2} \mathrm{H}_{5}+\mathrm{NH}_{3}=\mathrm{C}_{2} \mathrm{H}_{5} \mathrm{OH} \\
& +\mathrm{CH}_{3} \cdot \mathrm{C}\left\{\begin{array}{c}
\mathrm{H} \\
\mathrm{OC}_{6} \mathrm{H}_{5}
\end{array}\right\} \mathrm{CO} \cdot \mathrm{NH}_{2} \text {. }
\end{aligned}
$$

In kaltem Wasser ist das Amid unlöslich, aus heissem krystallisirt es in langen Nadeln aus; grosse Krystalle, wie sie sich bei der Bildung des Amids absetzten, entstanden nicht wieder; auch aus Alkohol und Aether, in welchen das Amid leicht löslich ist, krystallisirt es in Nadeln aus. Sein Schmelzpunkt liegt bei $130^{\circ}$.

Löst man das Amid in heisser Salzsäure, so scheiden sich beim Erkalten klare dünmblättrige Krystalle des salzsauren Oxyphenylpropionsäure-Amids aus. Aus einer Lösung in Schwefelsäure krystallisirt das schwefelsaure Amid. 


\section{Verhalten der Oxyphenylpropionsäure gegen Brom.}

$\mathrm{Zu}$ einer auf dem Wasserbade stark erhitzten wässrigen Lösung von Oxyphenylpropionsäure lässt man nach und nach so lange Bromwasser zutropfen, bis die Flüssigkeit sich gelb zu färben beginnt. Beim Erkalten scheidet sich dann eine grosse Menge weisser krystallinischer Flocken ab, welche, aus verdünntem Alkohol umkrystallisirt, feste, wasserklare, ziemlich grosse Nadeln geben.

I. 0,202 Grm. der im Vacuum getrockneten Substanz lieferten mit Kupferoxyd und vorgelegtem chromsatrem Blei verbrannt, 0,073 Grm. $\mathrm{H}_{2} \mathrm{O}$ und 0,326 Grm. $\mathrm{CO}_{2}$.

II. 0,215 Grm. derselben Substanz gaben, in geschlossener Röhre mit Salpetersäure und salpetersaurem Silber erhitzt, 0,166 Grm. AgBr.

Die Monobromoxyphenylpropionsäure:

$$
\mathrm{CH}_{3} \cdot \mathrm{C}\left\{\underset{\mathrm{OC}_{3} \mathrm{H}_{4} \mathrm{Br}}{\mathrm{H}}\right\} \mathrm{COOH}
$$

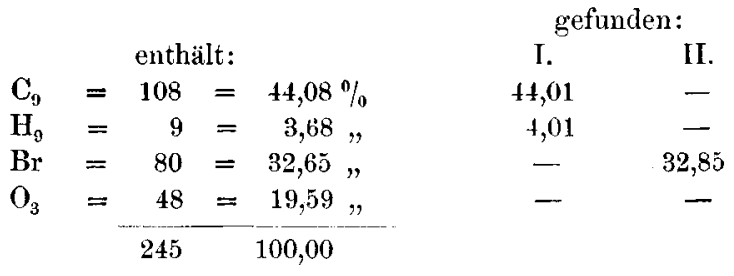

Die Krystalle, deren Schmelzpunkt bei 105-106 liegt, sind äusserst leicht löslich in Alkohol und Aether, in heissem Wasser lösen sie sich ziemlich schwer. Bei längerem Stehen an der Luft färben sie sich bräunlich, sind jedoch durch Umkrystallisiren aus Alkohol wieder leicht rein weiss zu erhalten. Aus einer alkoholischen Lösung scheidet sich die Süure auf Zusatz von Wasser in weissen Flocken ab.

Die in einer vorläufigen Mittheilung ${ }^{1}$ ) über Oxyphenylpropionsäure ausgesprochene Vermuthung, dass in der Monobromoxyphenylpropionsäure ein Wasserstoff des Phenyls durch Brom vertreten ist, wird dadurch bestätigt, dass durch concentrirte Natronlauge, selbst bei starkem Kochen, kein Brom aus der Verbindung herausgenommen wird. Das sich

') Dies. Journ. [2] 19, 175 . 
bei diesem Kochen mit Natronlauge bildende Natronsalz der Monobromoxyphenylpropionsäure: $\left.\mathrm{CH}_{3} . \mathrm{C}_{\mathrm{OC}_{6} \mathrm{H}_{4} \mathrm{Br}}^{\mathrm{H}}\right\} \mathrm{COONa}$ krystallisirt in langen glänzenden Nadeln, welche an der Luft sehr bald zerfliessen.

\section{Einwirkung von Eugenol auf Monochloressigsäure.}

$10^{\circ}$ Grm. Monochloressigsäure werden auf dem Wasserbade geschmolzen, $16 \mathrm{Grm}$. Eugenol zugefügt und in die Mischung nach und nach $30 \mathrm{Grm}$. Natronlauge (spec. Gew. 1,34) eingetragen. Die Flüssigkeit färbt sich dabei unter starker Erhitzung, welche bei weiterem Zusatz von Natronlauge sogar heftiges Sieden hervorbringt, immer dunkler. Man dampft, wenn alle Natronlauge eingetragen ist, auf dem Wasserbade weiter ein, bis die Masse ein breiiges Aussehen gewonnen. Nach dem Erkalten löst man in Wasser und trägt in die Lösung so lange Salzsäure ein, bis auf weiteren Zusatz keine milchige Trübung mehr erfolgt. Nach kurzer Zeit klärt sich die Flüssigkeit vollständig, und die neue Säure scheidet sich in feinen, glänzenden Nadeln ab. Zur vollständigen Reinigung der noch nach Eugenol riechenden Krystalle neutralisirt man sie, nachdem sie anf dem Saugfilter mit kaltem Wasser gut ausgewaschen sind, mit kohlensaurem Natron, Eugenol bleibt ungelöst; aus der filtrirten Lösung fällt man die Säure nochmals mit Salzsäure. Beim Umkrystallisiren der abgeschiedenen Masse aus heissem Wasser scheidet sich die neue Säure in über 2 Zoll langen, farblosen, prächtig seideschimmernden Nadeln ab.

Eine Analyse der bei $110^{\circ}$ getrockneten Substanz gab folgende Werthe:

$0,1780 \mathrm{Grm}$. lieferten, mit Kupferoxyd und Sauorstoff im offenen Rohre verbrannt, 0,1039 Grm. $\mathrm{H}_{2} \mathrm{O}$ und $0,4243 \mathrm{Grm} . \mathrm{CO}_{2}$.

Die Oxyeugenylessigsäure

$$
\begin{array}{lccc} 
& \text { enthält: } & \text { gefunden: } \\
\mathrm{C}_{12}=144=64,86 \% & 65,00 \\
\mathrm{H}_{14}=14=6,31 \% & 6,48 \\
\mathrm{O}_{4}=64=28,83 \% & - \\
& =622 & 100,00 &
\end{array}
$$


Die Säure schmilzt unter Wasser, ist aber in grösserer Menge desselben leicht löslich, noch leichter wird sie von Alkohol und Aether gelöst. Die Krystalle schmelzen unzersetzt bei $80--81^{\circ}$.

Die Einwirkung des Eugenols verläuft im Sinne der Gleichung:

$$
\begin{aligned}
& \mathrm{C}_{6} \mathrm{H}_{3}\left\{\begin{array}{l}
\mathrm{C}_{3} \mathrm{H}_{5} \\
\mathrm{OCH}_{3} \\
\mathrm{OH}
\end{array}+\mathrm{C}\left\{\begin{array}{l}
\mathrm{Cl}_{\mathrm{H}_{2}} \\
\text { Eugenol }
\end{array}\right\} \mathrm{COOH}+2 \mathrm{NaOH}\right. \\
& =\mathrm{NaCl}+2 \mathrm{H}_{2} \mathrm{O}+\mathrm{C}_{6} \mathrm{H}_{3}\left\{\begin{array}{l}
\mathrm{C}_{3} \mathrm{H}_{5} \\
\mathrm{OCH}_{3} \\
\mathrm{O} \\
\mathrm{C}_{\mathrm{H}_{2}}
\end{array}\right\} \mathrm{COONa} .
\end{aligned}
$$

Na-Salz der neuen Säure.

Durch Neutralisiren der Säure mit kohlensaurem Natron, Filtriren und Eindampfen erhält man ihr Natronsalz in klaren, ziemlich harten, spiessigen Krystallen, welche in Wasser sich leicht in der Kälte, in Alkohol aber erst beim Erhitzen lösen. Bei $110^{\circ}$ verliert das Salz vollständig sein Krystallwasser und schmilzt dann zu einer weissen glasigen Masse.

Analyse des zwischen Filtrirpapier gepressten Salzes:

I. $0,2960 \mathrm{Grm}$. Substanz verloren, bis $110^{\circ}$ erhitzt, $0,0298 \mathrm{Grm}$. $\mathrm{H}_{2} \mathrm{O}$.

II. 0,2662 Grm. entwässerte Substanz gaben nach dem Abrauchen mit Schwefelsäure $0,0815 \mathrm{Grm} . \mathrm{SO}_{4} \mathrm{Na}_{2}$.

Berechnet für:

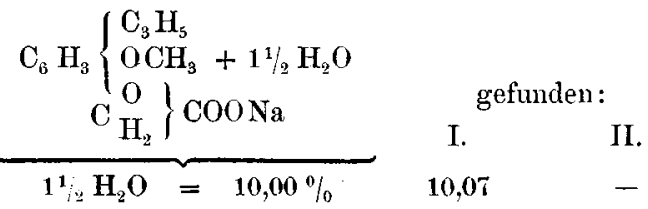

$$
\begin{aligned}
& \text { ber. auf trockne Substanz: }
\end{aligned}
$$

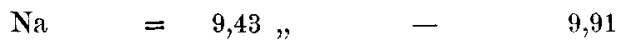

\section{Einwirkung von Thymol auf Monochloressigsäure.}

Auf dem Wasserbade schmilzt man 15 Grm. Thymol und $10 \mathrm{Grm}$. Monochloressigsäure und fügt dazu allmählich etwas mehr, als die äquivalente Menge Natronlauge (30 Grm. 


\section{Saarbach: Einwirkung von Phenolen}

vom spec. Gew. 1,34). Die Flüssigkeit erhitzt sich stark, und noch während der Operation scheiden sich aus ihr Krystallblättchen $a b$. Die unter gutem Umrühren ziemlich weit eingedampfte Masse wird in Wasser gelöst und mit Salzsäure versetzt, wobei sich die neue Säure in kleinen, weissen Narleln abscheidet, welche aber, selbst nach öfterem Umkrystallisiren aus heissem Wasser oder Alkohol, ihren starken Geruch nach Thymol nicht verlieren. Zur vollständigen Reinigung wurde durch Neutralisation mit kohlensaurem Natron das Natronsalz der nenen Säure dargestellt, und diese selbst mit Salzsäure aus der Lösung desselben abgeschieden. Beim Umkrystallisiren aus verdünntem Alkohol schieden sich lange, glasglänzende Nadeln $a b$, welche, wie die Analyse zeigt, aus vollkommen reiner Säure bestehen.

I. 0,1332 Grm. der bei $110^{\circ}$ getrockneten Substanz gaben, mit Kopferoxyd und Sauerstoff in offenen Rohre verbrannt, 0,1000 Grm. $\mathrm{H}_{2} \mathrm{O}$ und 0,3383 Grm. $\mathrm{CO}_{3}$.

II. $0,1751 \mathrm{Grm}$, auf dieselbe Weise verbrannt, lieferten 0,1224 Grm. $\mathrm{H}_{2} \mathrm{O}$ and 0,4430 Grm. $\mathrm{CO}_{2}$.

Die Oxythymylessigsïure: $\mathrm{C}_{6} \mathrm{H}_{3}\left\{\begin{array}{l}\mathrm{CH}_{3} \\ \mathrm{C}_{3} \mathrm{H}_{7} \\ \mathrm{O} \\ \left.\mathrm{C}_{\mathrm{H}_{2}}\right\}\end{array}\right.$

$$
\begin{array}{lcccc} 
& \text { enthält: } & \text { I. } & \text { II. } \\
\mathrm{C}_{12}=144=69,230_{0} & 69,26 & 68,99 \\
\mathrm{H}_{16}=16=7,69, & 8,34 & 7,77 \\
\mathrm{O}_{3}=48=23,08, & - & - \\
208 & 100,00 & &
\end{array}
$$

Die Säure ist in kaltem Wasser sehr wenig, in heissem Wasser, Alkohol und Aether leicht löslich. Die Krystalle schmelzen bei $148^{\circ}$, bei $132^{\circ}$ erstarren sie wieder zu einer weissen krystallinischen Masse.

Die Bildung der Säure verläuft folgendermaassen:

$$
\begin{aligned}
& \mathrm{C}_{6} \mathrm{H}_{3}\left\{\begin{array}{l}
\mathrm{CH}_{3} \\
\mathrm{C}_{3} \mathrm{H}_{7} \\
\mathrm{OH}
\end{array}+\mathrm{C}\left\{\begin{array}{l}
\mathrm{Cl}_{\mathrm{H}_{2}} \\
\text { Thymol }
\end{array}\right.\right. \\
& =\mathrm{COOH}+2 \mathrm{NaOH}
\end{aligned}
$$


Die Oxythymylessigsäure lässt sich leicht destilliren und schiesst in der gekühlten Vorlage in langen, spitzen wasserklaren Nadeln an. Eine Verbrennung des Destillates, welches einen schwach brenzlichen Geruch angenommen, gab sowohl für Kohlenstoff, als für Wasserstoff zu hohe Werthe $(\mathrm{C}=70,03, \mathrm{H}=8,26 \%$ ); dieses Resultat lässt auf eine theilweise Zersetzung, auf einen geringen Gehalt an Thymol, schliessen.

\section{Einwirkung von Phenol auf Dibrombernsteinsäure.}

Lässt man der Giacosa'schen Darstellungsweise analog 10 Grm. Phenol, 14 Grm. Dibrombernsteinsäure und 30 Grm. Natronlauge auf einander einwirken, löst den gewonnenen Krystallbrei in Wasser und versetzt die Lösung mit Salzsäure, so ist, selbst nach längerem Stehen, die Abscheidung eines Körpers nicht zu bemerken. Die mit Salzsäure versetzte Lösung wurde deshalb eingedampft und der erhaltene krystallinische Rückstand mit Alkohol ausgezogen; ungelöst blieb eine weisse Masse aus Würfelchen von Chlornatrium und Bromnatrium bestehend. Der alkoholische Auszug hinterliess nach längerem Absaugen unter dem Exsiccator statt der erwarteten Krystalle einen braun gefärbten, syrupartigen Rückstand von angenehm ätherischem Geruch. Beim Waschen mit Wasser setzt er sich als schwer flüssige Schicht am Boden $a b$; bei der Destillation mit Wasserdämpfen geht er als ganz klare Flüssigkeit über. Eine Probe der Substanz, mit Kalk geglüht und mit salpetersaurem Silber behandelt, zeigt, dass sie noch Brom, sogar in ziemlicher Menge, enthielt; die quantitative Bestimmung des Kohlenstoffs, Wasserstoffs und des Broms gaben keinen Aufschluss.

Eine weitere Reinigung der Substanz war wegen der geringen Menge, welche ich erhalten hatte, nicht gut vorzunehmen; ihr Gehalt an Brom lässt jedentalls erkennen, dass die Einwirkung des Phenols auf Dibrombernsteinsäure bei der eben beschriebenen Behandlung nicht in der gewünschten Weise verläuft; ich gedenke deshalb noch den Versuch zu machen, die Natronsalze beider Körper, in analoger Weise Jourual f. prakt. Chemie [2] Bd. 21. 
wie bei der Bildung der Oxyphenylpropionsäure, auf einander einwirken zu lassen, und hoffe ich darüber nächstens berichten zu können.

\section{Einwirkung von Orcin auf Monochloressigsäure.}

Die glatte Umsetzung von einbasischen Phenolen mit Monochloressigsäure regte die Frage an, ob auch zweibasische Phenole ein ähnliches Verhalten zeigen. Die Versuche, welche mit dem Orcin angestellt wurden, haben ein günstiges Resultat ergeben: es entsteht eine zweibasische Säure, welche in ihren Eigenschaften grosse Aehnlichkeit mit den vorher beschriebenen erkennen lässt.

Das beste Verfahren zu ihrer Darstellung ist folgendes:

$\mathrm{Zu}$ einer auf dem Wasserbade geschmolzenen Mischung von $62 \mathrm{Grm}$. Orcin und $100 \mathrm{Grm}$. Monochloressigsäure fügt man nach und nach $540 \mathrm{Grm}$. Natronlauge $(31 \%)$. Die eintretende Reaction ist sehr heftig, bald geräth die Flüssigkeit in's lebhafteste Sieden, und wenn ungefähr $3 / 4$ der Natronlauge eingetragen ist, erstarrt die ganze Masse $\mathrm{zu}$ einem schwach gelblich gefürbten Brei, welchem man unter gutem Umrühren noch den Rest der Natronlauge zusetzt. Löst man die erkaltete Masse in Wasser und säuert mit Salzsäure an, so scheidet sich aus der Lösung eine grosse Menge weisser Körnchen ab, welche sich nach kurzer Zeit zu Boden setzen. Nachdem sie auf dem Saugfilter einige Male mit kaltem Wasser ausgewaschen sind, werden sie aus heissem Wasser umkrystallisirt und stellen dann weisse Flocken dar, welche, wie das Mikroskop zeigt, aus zarten spitzen Nädelchen bestehen.

Die Einwirkung des Orcins verläuft gemäss der Gleichung:

$$
\begin{aligned}
& \mathrm{C}_{6} \mathrm{H}_{3}\left(\mathrm{CH}_{3}\right)\left\{\begin{array}{l}
\mathrm{OH} \\
\mathrm{OH}
\end{array}+2 \mathrm{C}\left\{\begin{array}{l}
\mathrm{Cl} \\
\mathrm{H}_{3}
\end{array}\right\} \mathrm{COOH}+4 \mathrm{NaOH}\right. \\
& \left.=2 \mathrm{NaCl}+4 \mathrm{H}_{2} \mathrm{O}+\mathrm{C}_{6} \mathrm{H}_{3}\left(\mathrm{CH}_{3}\right) \underset{\mathrm{C}_{\mathrm{H}_{2}}}{\mathrm{C}_{2}} \begin{array}{l}
\mathrm{O} \\
\mathrm{O}
\end{array}\right\} \mathrm{COONa}
\end{aligned}
$$


Bei der eben besprochenen Darstellung sind Monochloressigsäure und Natronlauge in kleinem Ueberschusse angewandt; nimmt man der Gleichung entsprechende Mengen, so erhält man immer ein noch stark gelb gefärbtes Product, welches selbst durch öfteres Neutralisiren mit kohlensaurem Kalk und Soda und nachheriges Wiederausfällen, Umkrystallisiren und Reinigen mit Thierkohle nicht weiss zu bekommen ist. Die Ursache davon liegt wahrscheinlich in der theilweisen Zersetzung der Monochloressigsäure beim Kochen mit Natronlauge.

Zur schnelleren Reingewinnung der vorher beschriebenen Säuren ist es empfehlenswerth, Monochloressigsäure und Natronlauge im Ueberschuss zuzufügen.

Die Analysen des bei $110^{\circ}$ getrockneten reinen Körpers gaben folgende Resultate:

I. 0,1212 Grm., mit Kupferoxyd und Sauerstoff im offnen Rohre verbrannt, lieferten $0,0583 \mathrm{Grm} . \mathrm{H}_{2} \mathrm{O}$ und $0,2429 \mathrm{Grm}$. $\mathrm{CO}_{2}$.

II. 0,1121 Grm., auf dieselbe Weise verbrannt, lieferten 0,0540 Grm. $\mathrm{H}_{2} \mathrm{O}$ und $0,2258 \mathrm{Grm} . \mathrm{CO}_{2}$.

$$
\begin{aligned}
& \text { Dioxyorcyldiessigsäure: } \mathrm{C}_{6} \mathrm{H}_{3}\left(\mathrm{CH}_{3}\right)\left\{\begin{array}{l}
\mathrm{C}_{\mathrm{O}}^{\mathrm{H}_{2}} \\
\mathrm{O} \\
\mathrm{O} \\
\mathrm{H}_{2}
\end{array}\right\} \mathrm{COOH} \\
& \begin{array}{rlrr} 
& \multicolumn{2}{c}{\text { enthält: }} & \multicolumn{2}{c}{\text { gefunden: }} \\
\mathrm{C}_{11}=132=55,00 \% & \text { I. } & \text { II. } \\
\mathrm{H}_{12}=12=5,00 \% & 54,66 & 54,95 \\
\mathrm{O}_{6}= & \mathbf{5 6}=40,00 \% & - & 5,35 \\
\hline \mathbf{2 4 0} & 100,00 & & -
\end{array}
\end{aligned}
$$

Sie ist eine starke zweibasische Säure, welche Kohlensäure aus ihren Salzen leicht austreibt. In kochendem Wasser ist sie ziemlich, in Aether und Alkohol leicht löslich, Umkrystallisiren aus Alkohol ist jedoch deshalb nicht rathsam, weil sie sich damit schon beim Stehen in der Kälte ätherificirt. Ihr Schmelzpunkt liegt bei 216-217 .

Auf dem Platinblech schmilzt sie zu einer dunklen Flüssigkeit, welche bei weiterem Erhitzen sich unter vollständiger Verflüchtigung entzündet. 


\section{Saarbach: Einwirkung von Phenolen}

Eisenchlorid erzeugt in einer Lösung von Dioxyorcyldiessigsäure einen röthlich gelben, flockigen Niederschlag, welcher sich beim Erhitzen nicht merklich verändert.

Um den Zusammenhang dieser Säure mit der Oxyphenylessigsäure und Oxyphenylpropionsäure klar hervortreten zu lassen, wählte ich für sie den ganz analog gebildeten Namen Dioxyorcyldiessigsäure, wobei unter Dioxyorcyl das zweibasische Radical $\left(\mathrm{C}_{6} \mathrm{H}_{3}\left(\mathrm{CH}_{3}\right)\left\{\begin{array}{l}0 \\ \mathrm{O}\end{array}\right)^{\prime \prime}\right.$ zu verstehen ist.

\section{Salze der Dioxyorcyldiessigsäure.}

Das Natronsalz wird durch Neutralisiren der Säure mit kohlensaurem Natron, Filtriren und Eindampfen in warzenförmigen gelblichen Gebilden erhalten, welche durch öfteres Umkrystallisiren allmählich weiss werden.

I. 0,3984 Grm. Iufttrockner Substanz verloren beim Erhitzen bei $150^{\circ} 0,0634$ Grm. $\mathrm{H}_{2} \mathrm{O}$.

II. 0,3350 Grm. entwässerte Substanz gaben nach dem Abrauchen mit Schwefelsäure 0,1623 Grm. $\mathrm{SO}_{4} \mathrm{Na}_{2}$.

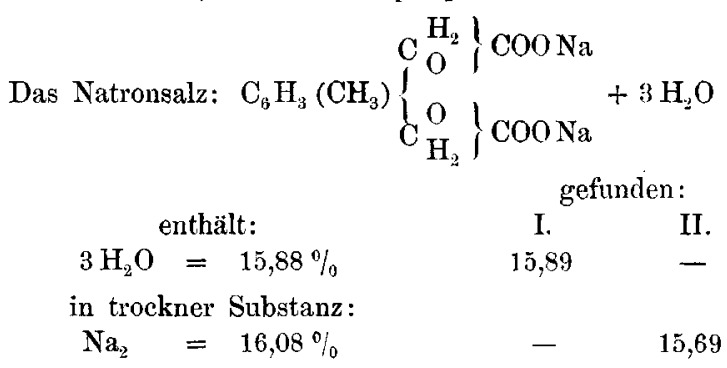

In Wasser ist das Natronsalz ausserordentlich leicht löslich und erstarrt erst dann krystallinisch, wenn das Wasser durch Abdampfen fast vollständig verjagt ist; in Alkohol löst es sich erst beim Erhitzen und krystallisirt aus ihm in prachtvollen, sternförmig gruppirten Nadeln aus. ${ }^{1}$ )

1) Ein Versuch, das Natronsalz der Dioxyorcyldiessigsäure zu elektrolysiren, wurde angestellt, um zu untersuchen, ob dieses dabei unter Abspaltung von $2 \mathrm{CO}_{2}$ und $2 \mathrm{Na}$ einen Körper hinterlässt, den man am besten als Aethylenoxydhydrat ansieht, dessen $2 \mathrm{H}$ durch das zweiwerthige Orcyl vertreten sind: 
Das Kalisalz, in analoger Weise wie das Natronsalz dargestellt, krystallisirt aus wässriger Lösung in blumenkohlartigen Gebilden, welche sich bei langsamem Abscheiden zu strahligen Aggregaten vereinigen. Es ist in heissem Alkohol wenig löslich und schiesst daraus in kurzen, klaren Nadeln an. Beim Erhitzen im Luftbade bräunt es sich; bei einigen Versuchen verlor es verschiedene Mengen von Wasser.

0,5005 Grm. luftrockner Substanz gaben, mit Schwefelsäure abgeraucht, $0,2355 \mathrm{Grm} . \mathrm{SO}_{4} \mathrm{~K}_{2}$.

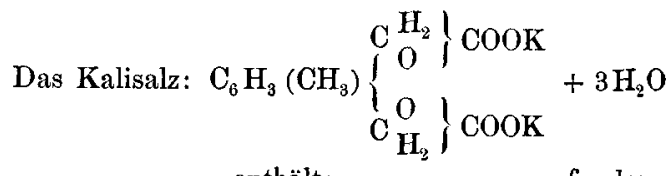

$$
\begin{array}{ll}
\mathrm{K}_{2}=21,08 \% & 21,09
\end{array}
$$

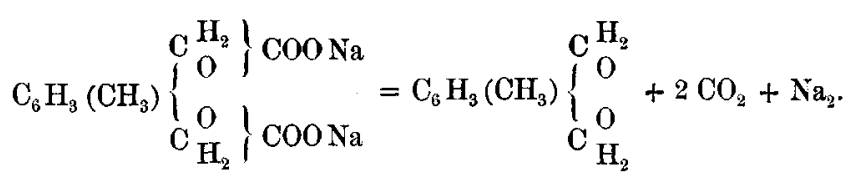

Leitet man einen durch 6 Bunsen'sche Elemente erzeugten galvanischen Strom durch die concentrirte Lösung des dioxyorcyldiessigsauren Natrons, so entweicht ein Gas, welches Kalkwasser sofort trübt und, über Natronlauge aufgefangen, an der Flamme unter heftigem Knall explodirt.

Die Lösung färbte sich während des Durchgehens des Stromes immer dunkler, die Abscheidung eines Oeles ist jedoch nicht wahrznnehmen; beim Abdampfen der Lösung scheidet sich unverändertes Natronsalz wieder ab; durch Ausschütteln mit Aether wird der Lösung Nichts entzogen.

Um zu prüfen, ob sich in den entweichenden Gasen ausser Kohlensäure ein kohlenstoffhaltiges Gas befand, wurden sie, nachdem sie durch Natronlauge und eine alkalische Lösung von Pyrogallussäure gegangen, durch eine Röhre mit glühendem Kupferoxyd geleitet; eine Trübung des Kalkwassers, durch welches nachher das Gas entwich, war selbst nach längerem Durchleiten nicht zu bemerken.

Die übrige Lösung wurde mit Salzsäure versetzt und dann auch das Filtrat der abgeschiedenen Säure mit Aether ausgeschüttelt, auch hieraus nahm derselbe Nichts auf.

Es ist wahrscheinlich, dass die Zersetzung durch den elektrischen Strom bei der eventuellen Bildung des vorerwähnten Körpers nicht stehen bleibt, sondern auch dieser weiter zerlegt wird. 
Dioxyorcyldiessigsaurer Kalk scheidet sich bei raschem Eindampfen in dünnen Blättern, bei langsamem Abdunsten in kleinen, harten Krystallen aus. Er krystallisirt mit 2 Molekülen $\mathrm{H}_{2} \mathrm{O}$.

I. 0,3166 Grm. lufttrockner Substanz verloren beim Erhitzen bis $120^{\circ} 0,0345$ Grm. $\mathrm{H}_{2} \mathrm{O}$.

II. 0,2812 Grm. entwässerte Substanz gaben, mit Schwefelsäure abgeraucht, 0,1355 Grm. $\mathrm{SO}_{4} \mathrm{Ca}$.

III. 0,0895 Grm. derselben Substanz lieferten, mit Kupferoxyd und Sauerstoff im offenen Rohre verbrannt, 0,0320 Grm. $\mathrm{H}_{2} \mathrm{O}$ and 0,1548 Grm. $\mathrm{CO}_{2}$.

$$
\begin{aligned}
& \text { Berechnet auf: }
\end{aligned}
$$

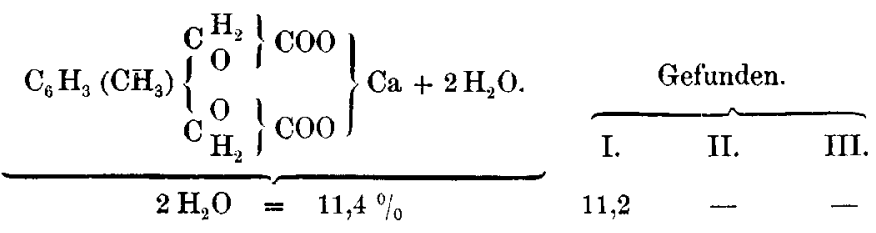

$$
\begin{aligned}
& \begin{array}{lrrrrr}
\mathrm{C}_{11}=132 & =47,48 \% & - & - & 47,17 \\
\mathrm{H}_{10}=10=3,61, & - & - & 3,97 \\
\mathrm{O}_{6}=96=34,53 \% & - & - & - \\
\mathrm{Ca} & =40=14,38 \% & & - & 14,17 & - \\
\hline 278 & 100,00 & & &
\end{array}
\end{aligned}
$$

Das Kalksalz ist in Wasser ziemlich leicht, in Alkohol schwer löslich.

Bei der trocknen Destillation schwärzt es sich durch die ganze Masse; zuletzt, bei starker Erhitzung, geht ein braunes, brenzlich riechendes, zähflüssiges Oel über, welches weder zum Krystallisiren gebracht, noch überhaupt gereinigt werden konnte.

Das dioxyorcyldiessigsaure Kupfer fällt bei $\mathrm{Zu}$ satz von Kupfervitriol zur wässrigen Lösung des Natronsalzes als schmutzig grün gefärbter, dickflockiger Niederschlag aus.

Das Bleisalz, durch Neutralisation der Säure mit kohlensaurem Blei erhalten, krystallisirt in dünnen, glasglänzenden Blättchen. 
Dioxyorcyldiessigsäure-A ethyläther.

Schon bei dem Versuche, Dioxyorcyldiessigsäure aus Alkohol umzukrystallisiren, bildet sich, wie erwähnt, der Aether. Die beste Ausbeute wird erhalten, wenn man durch eine alkoholische Lösung der Säure Salzsäure leitet, noch einige Zeit im Wasserbade stark erhitzt und dann das erhaltene Product in Wasser giesst. Es scheidet sich dabei ein schweres, sehr zähes Oel ab, welches bei der Reinigung mit Wasserdämpfen ein Destillat liefert, das zwar einen angenehm ätherischen Geruch angenommen hat, aus welchem sich aber selbst nach längerer Zeit Nichts abscheidet. Bei dem Versuche, das zurückgebliebene Oel mit Chlorcalcium zu trocknen, erstarrte die ganze Masse zu einem festen, krystallinischen Brei, welcher beim Destilliren im Vacuum ebenfalls kein reines Product gab. Der Aether wurde deshalb aus der Chlorcalciumverbindung mit Wasser wieder abgeschieden, mit Aether ausgeschüttelt, die ätherische Lösung nochmals mit Chlorcalcium entwässert, filtrirt und der zur Lösung verwandte Aether unter dem Exsiccator verdunstet. Es hinterblieb eine schmierige, braun gefärbte Masse, welche, auf eine Gypsplatte gebracht, sich schnell entfärbte und unter dem Exsiccator vollständig erstarrte. Beim Umkrystallisiren aus absolutem Alkohol erhält man daraus eine schneeweisse Masse.

I. $0,0855 \mathrm{Grm}$. der bei $100^{\circ}$ getrockneten Substanz lieferten, im offnen Rohre verbrannt, $0,0543 \mathrm{Grm} . \mathrm{H}_{2} \mathrm{O}$ und 0,1899 Grm. $\mathrm{CO}_{2}$.

II. 0,0792 Grm. Substanz gaben 0,0495 Grm. $\mathrm{H}_{2} \mathrm{O}$ und 0,1754 Grm. $\mathrm{CO}_{2}$.

Diese Werthe führen zu der Zusammensetzung:

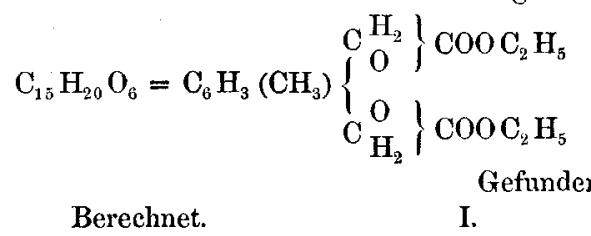

$\begin{array}{lrrrr}\mathrm{C}_{15}= & 180= & 60,81 & 60,57 & 60,39 \\ \mathrm{H}_{20}= & 20= & 6,76 & 7,06 & 6,83 \\ \mathrm{O}_{6}= & 96= & 32,43 & & -\end{array}$


168 Saarbach: Einwirkung von Phenolen

Der Dioxyorcyldiessigsäure-Aethyläther stellt eine flockige Krystallmasse dar, welche aus kleinen Nädelchen besteht, deren Schmelzpunkt bei $107^{\circ}$ liegt. Nach dem Schmelzen erstarrt der Aether zu warzenförmigen Gebilden. In Wasser ist er unlöslich, in Alkohol und Aether leicht löslich; aus diesen Lösungen wird er durch Wasser als Oel abgeschieden.

Ueberschichtet man dieses mit concentrirtem wässrigem Ammoniak, so scheiden sich nach einiger Zeit körnige Blättchen des

Dioxyorcyldiessigsäure-Amids

aus. Aus Wasser umkrystallisirt, mit Alkohol und Thierkohle gereinigt, stellt es zarte, weisse Flocken dar, welche selbst unter dem Mikroskop ein krystallinisches Gefüge nicht erkennen lassen.

Eine Stickstoffbestimmung der bei $110^{\circ}$ getrockneten Masse ergab : Cem. N.

$0,2242 \mathrm{Grm}$. Substanz lieferten bei $758 \mathrm{Mm}$. Bar. und $20^{\circ} 22,5$

$$
\begin{array}{cc}
\text { Berechnet auf das Amid: } & \text { Gefunden: } \\
\mathrm{N}=11,76 \% & 11,46
\end{array}
$$

Die Umwandlung des Aethers in das Amid veranschaulicht die Gleichung:

$$
\begin{aligned}
& \mathrm{C}_{6} \mathrm{H}_{3}\left(\mathrm{CH}_{3}\right)\left\{\begin{array}{l}
\mathrm{C}^{\mathrm{H}_{2}} \\
\mathrm{O}
\end{array}\right\} \mathrm{COOC}_{2} \mathrm{H}_{5}+2 \mathrm{NH}_{3} \\
& \left.=2 \mathrm{C}_{2} \mathrm{H}_{5} \mathrm{OH}+\mathrm{C}_{6} \mathrm{H}_{3}\left(\mathrm{CH}_{3}\right) \underset{\mathrm{C}_{\mathrm{H}_{2}}}{\int_{\mathrm{O}}^{\mathrm{O}}} \begin{array}{l}
\mathrm{H}_{2} \\
\mathrm{O}
\end{array}\right\} \mathrm{CO} \cdot \mathrm{NH}_{2} . \mathrm{NH}_{2} .
\end{aligned}
$$

Löst man das Amid in heisser Salzsäure, so scheidet sich beim Erkalten das salzsaure DioxyorcyldiessigsäureAmid in zarten, strahlig gruppirten Nädelchen ab.

$$
\begin{aligned}
& \text { Verhalten der Dioxyorcyldiessigsäure gegen } \\
& \text { Salpetersäure. }
\end{aligned}
$$

Kalte Salpetersäure bewirkt beim Zusammenbringen mit Dioxyorcyldiessigsäure nur eine schwache Dunkelfärbung 
der letzteren; erwärmt man aber etwa $100 \mathrm{Grm}$. Salpetersäure (spec. Gew. 1,12) auf dem Wasserbade und trägt nach und nach $20 \mathrm{Grm}$. Dioxyorcyldiessigsäure ein, so beginnt die Masse beim jedesmaligen Zufügen unter Ausstossung rother Dämpfe auf's Heftigste zu sieden und zu stossen. Um Ueberschäumen zu verhüten, muss man das Becherglas vom Wasserbade herunternehmen. Nach Beendigung der Reaction entsteht eine schön carminroth gefärbte Lösung, welche bei jedesmaligem Eintragen der Säure, was nur in kleinen Portionen geschehen darf, sich zuerst dunkel graugrün färbt, bei schwacher Erwärmung aber wieder in's lebhafteste Sieden geräth, und zuletzt die schön gefärbte klare Lösung giebt, aus welcher sich beim Erkalten ein rother pulveriger Niederschlag in ziemlicher Menge absetzt. Man gewinnt diesen noch besser, wenn man nach Beendigung der Reaction die ganze Masse in eine grössere Quantität kaltes Wasser giesst, welches Alles, mit Ausnahme des oben erwähnten rothen Körpers, löst, den man durch gutes Auswaschen mit Wasser und Umkrystallisiren aus Alkohol unter Zusatz von Thierkohle reinigt.

Er stellt dann ein hellrothes Pulver dar, welches, bei $100^{\circ}$ getrocknet, der Analyse unterworfen wurde.

I. 0,0912 Grm. Substanz, mit Kupferoxyd und vorgelegten Kupferspiralen verbrannt, lieferten $0,0338 \mathrm{Grm} . \mathrm{H}_{2} \mathrm{O}$ und $0,1544 \mathrm{Grm} . \mathrm{CO}_{2}$.

II. 0,2501 Grm. Substanz gaben $11 \mathrm{Ccm}$. N (T. $=18^{\circ} ;$ Bar. $=$ $749 \mathrm{Mm}$.).

Diese Werthe führen zu der Zusammensetzung der Mononitrodioxyorcyldiessigsäure:

$$
\begin{aligned}
& \mathrm{C}_{11} \mathrm{H}_{11} \mathrm{NO}_{3}=\mathrm{C}_{6} \mathrm{H}_{2}\left\{\begin{array}{c}
\mathrm{NO}_{2} \mathrm{C}_{\left(\mathrm{CH}_{3}\right)}^{\mathrm{H}_{2}} \mathrm{C}_{\mathrm{C}}^{\mathrm{O}} \\
\mathrm{C}_{\mathrm{H}_{2}}
\end{array}\right\} \mathrm{COOH} . \\
& \mathrm{C}_{11}=132=46,32 \\
& \mathrm{H}_{11}=11=3,86 \\
& \mathrm{~N}=14=4,91 \\
& \text { Gefunden. } \\
& O_{8}=\frac{128=44,91}{285 \quad 100,00}
\end{aligned}
$$




\section{Saarbach: Einwirkung von Phenolen}

In Alkohol und Aether ist die Mononitrodioxyorcyldiessigsäure leicht löslich, bei $140^{\circ}$ schmilzt sie zu einer rothen Flüssigkeit, auf dem Platinblech schmilzt sie unter Schwärzung, bei stärkerem Erhitzen verflüchtigt sie sich vollständig.

Die dem blossen Auge amorph erscheinende Masse erweist sich unter dem Mikroskop als aus sehr kleinen, spitzen Pyramiden zusammengesetzt.

Dampft man das bei der Darstellung dieser Säure erhaltene Filtrat stark ein und lässt es dann längere Zeit unter dem Exsiccator stehen, so erhält man einen schwarzen Syrup, in welchem sich darin herumschwimmende Kryställchen erkennen lassen. Bringt man diese Masse, wenn sie unter dem Exsiccator nicht mehr weiter eintrocknet, auf eine Gypsplatte, so hinterbleibt ein schwach gelblich gefärbtes Produkt, welches nach Umkrystallisiren aus Alkohol völlig farblose monokline Täfelchen bildet.

0,2407 Grm. Substanz lieferten $11,5 \mathrm{Ccm}$. N-Gas bei $750 \mathrm{Mm}$. Bar. und $23^{\circ}$.

Eine Verbrennung mit der noch übrigen Substanz verunglückte leider, die Stickstoffbestimmung macht es jedoch wahrscheinlich, dass dieser Körper eine mit der vorhergehend beschriebenen isomere Mononitrodioxyorcyldiessigsäure ist.

Berechnet für:

Dinitrodioxyorcyldiessigsäure:

$$
\mathrm{N}=8,48 \%
$$

Mononitroorein:

$\mathrm{N}=8,22 \%$

Mononitrodioxyorcyldiessigsäure:

$$
\mathrm{N}=4,91 \%
$$

In Alkohol, Aether und Wasser ist der Körper leicht löslich.

Die Resultate der vorstehenden Untersuchung sind, kurz zusammengefasst, folgende:

Phenol und phenolartige Körper wirken auf halogensubstituirte Fettsäuren im Beisein von Natronlauge derart 
ein, dass die Halogenatome durch die Oxy (phenyl- etc.) radicale ersetzt werden. Es entstehen so neue Säuren von sehr grosser Beständigkeit: die Oxyphenylpropionsäure, die Oxythymylessigsäure und die Oxeugenylessigsäure. Als erstes Glied dieser Säuren ist die von W. Heintz schon dargestellte Phenoxacetsäure anzusehen.

Zweibasische Phenole, wie Orcin, wirken in analoger Weise: durch Umsetzung mit 2 Molekülen Monochloressigsäure entsteht die zweibasische Dioxyorcyldiessigsäure.

Die Einwirkung des Phenols auf Dibrombernsteinsäure hat kein genügendes Resultat ergeben.

Sämmtliche hier beschriebene Säuren krystallisiren gut; Kohlensäure wird von ihnen schon in der Kälte ausgetrieben, die dabei sich bildenden Salze sind nicht sehr krystallisationsfähig, einige zerfliessen schon an der Luft.

Oxyphenylpropionsäure und Dioxyorcyldiessigsäure ätherificiren sich schon beim Stehen ihrer alkoholischen Lösung.

Durch Ueberschichtung der Aether mit Ammoniak entstehen sehr leicht die Amide der Säuren.

Auf Oxyphenylpropionsäure wirkt Brom einfach substituirend ein unter Bildung der Monobromoxyphenylpropionsäure.

Durch Behandlung mit Salpetersäure entstehen aus Dioxyorcyldiessigsäure zwei isomere Mononitrodioxyorcyldiessigsäuren.

Leipzig, Kolbe's Laboratorium, December 1879. 